\title{
Unusual Angular and Temperature Dependence of the Upper Critical Field in UPt
}

\author{
B. S. Shivaram ${ }^{(a)}$ and T. F. Rosenbaum \\ The James Franck Institute and the Department of Physics, The University of Chicago, Chicago, Illinois 60637 \\ and \\ D. G. Hinks \\ Materials Science and Technology Division, Argonne National Laboratory, Argonne, Illinois 60439
}

(Received 2 June 1986)

\begin{abstract}
We report measurements of the upper critical field, $H_{c 2}$, inclined at various angles with respect to the $c$ axis in the heavy-fermion superconductor $\mathrm{UPt}_{3}$. The angular anisotropy observed near $T_{c}=0.53 \mathrm{~K}$ cannot be explained quantitatively by presently available theoretical expressions which consider either isotropic or anisotropic pairing. In addition, we find that the anisotropy apparently disappears at $T \sim 200 \mathrm{mK}$, only to reemerge at lower temperatures with an opposite sense. We have also studied $H_{c 2}$ in the basal plane of this hexagonal crystal and find no angular dependence within the limits of our measurements.
\end{abstract}

PACS numbers: 74.60.-w, 74.30.Gn, 74.70.Lp

The heavy-fermion superconductor $\mathrm{UPt}_{3}$ has attracted considerable recent attention because of the wide range of unusual physical properties its exhibits. ${ }^{1}$ It is a member of a class of cerium- and uranium-based compounds where magnetic, impurity, mixed-valence, and superconducting effects all compete. Although the effective mass of the electrons in $\mathrm{UPt}_{3}$ is $\sim 200$ times the bare mass, it goes superconducting at $T \sim 0.5 \mathrm{~K}$. The presence of the $T^{3} \ln T$ term in its specific heat at low temperatures, ${ }^{1}$ measurements of the upper critical field ${ }^{2}$ and thermal conductivity, ${ }^{3}$ and the power-law behavior with temperature of the attenuation of longitudinal ultrasound ${ }^{4}$ have led to the speculation that the superconductivity in $\mathrm{UPt}_{3}$ involves anisotropic pairing. Recent measurements of the attenuation of transverse sound ${ }^{5}$ present further evidence of this anisotropy and suggest that the superconducting state is a polar phase ${ }^{6}$ with a line of zeros in the basal plane. An additional method of characterizing the superconducting state is to look for similar anisotropic effects in the upper critical field. If the superconducting state consists of quasiparticles paired in an $l>0$ angular momentum state, then one expects a spontaneous anisotropy which could be different from the underlying crystal symmetry. ${ }^{7,8}$

Measurements to date of the upper critical field, $H_{c 2}$, in single crystals of $\mathrm{UPt}_{3}$ have been confined to the major crystal axes and only extend down to $T \sim 150 \mathrm{mK} .^{2}$ In this paper, we report new results on the angular dependence of $H_{c 2}$ between the $c$ axis and the basal plane, down to $T=10 \mathrm{mK}$. We also report measurments for various directions in the basal plane for which no anisotropy is observed.

The present experiments were performed on two single crystals of $\mathrm{UPt}_{3}$ which came from the same batch as the samples in Ref. 5. The crystals were mounted on rotatable copper holders which were bolted on to the sample slug of a top-loading dilution refrigerator. Current and voltage leads were attached by means of silver epoxy. Typical sample dimensions were $0.15 \times 0.5 \times 2.0 \mathrm{~mm}^{3}$, and the long axis was always held perpendicular to the magnetic field so as to minimize the effect of demagnetization factors on the intrinsic rotational anisotropy. We estimate that the demagnetization correction is always $\leq 0.2 \%$. Current densities of less than $0.2 \mathrm{~A} / \mathrm{cm}^{2}$ were employed in measurements with a transformer-coupled bridge at 17 Hz. Data were collected by stepping of the field in small steps at constant temperature. The 10\%-90\% transition width was $\sim 150 \mathrm{Oe}$, independent of applied field.

The various parameters we measure for the two samples are shown in Table I along with their comparison to the values given by other groups. The variation of the resistivity below $1 \mathrm{~K}$ follows the form $\rho=\rho_{0}+A T^{n}$ with $n=1.6 \pm 0.1$, in agreement with the results obtained by Chen et l. $^{2}$ We deduce an electron mean free path $l=1800 \AA$ from the value of $\rho_{0}$.

We plot in Fig. 1 critical fields parallel and perpendicular to the hexagonal $c$ axis, $H_{c 2 \|}$ and $H_{c 21}$, respectively. The slopes at $T_{c}$ are the largest yet observed for $\mathrm{UPt}_{3}$ (see Table I), from which we determine a superconducting coherence length $\xi=110 \AA$. In addition, the positive curvature observed by Chen et al. ${ }^{2}$ for $H_{c 2 \perp}$ is less pronounced in Fig. 1 and is completely absent in $H_{c 2 \| 1}$. At lower temperature, $H_{c 211}$ and $H_{c 21}$ not only approach each other, but cross at $T \approx 200$ mK. Although $\left[d H_{c 2 \cdot 1} / d T\right]_{T_{c}}$ is only $60 \%$ of $\left[d H_{2 \|} /\right.$ $d T]_{T_{c}}, \quad H_{c 2 \perp}(T \rightarrow 0)$ is enhanced by $23 \%$ over $H_{c 2 \|}(T \rightarrow 0)$.

It has been suggested ${ }^{9}$ that the disapperance of the anisotropy, as must occur for critical field curves 
TABLE I. Properties of the two single crystals of $\mathrm{UPt}_{3}$ described in this paper, compared with the results of other groups (Ref. 2). The slopes near $T_{c}$ and the upper critical field as $T \rightarrow 0$ are the largest yet reported. Columns 6 and 7 come from fitting of the normal-state resistivity by $\rho=\rho_{0}+A T$ (Refs. 1 and 6). We also note the anisotropy in $\rho$ at room temperature.

\begin{tabular}{|c|c|c|c|c|c|c|c|c|c|c|}
\hline \multirow[b]{2}{*}{ Sample } & \multirow{2}{*}{$\begin{array}{l}\text { Current } \\
\text { density } \\
J\left(\mathrm{~A} / \mathrm{cm}^{2}\right)\end{array}$} & \multirow{2}{*}{$\begin{array}{r}T_{c} \\
(\mathrm{~K})\end{array}$} & \multirow{2}{*}{$\begin{array}{l}\rho(300 \mathrm{~K}) \\
(\mu \Omega-\mathrm{cm})\end{array}$} & \multirow[t]{2}{*}{$\frac{\rho(300 \mathrm{~K})}{\rho(1 \mathrm{~K})}$} & \multirow{2}{*}{$\begin{array}{c}\rho_{0} \\
(\mu \Omega-\mathrm{cm}) \\
\end{array}$} & \multirow[t]{2}{*}{$\left(\begin{array}{c}A \\
\frac{\mu \Omega-\mathrm{cm}}{\mathrm{K}^{1.6}}\end{array}\right.$} & \multicolumn{2}{|c|}{$\begin{array}{c}{\left[d H_{c 2} / d T\right]_{T_{c}}} \\
(\mathrm{kOe} / \mathrm{K})\end{array}$} & \multicolumn{2}{|c|}{$\begin{array}{c}H_{c}(T=0) \\
(\mathrm{kOe})\end{array}$} \\
\hline & & & & & & & II & $\perp$ & $\|$ & $\perp$ \\
\hline 1 & $\begin{array}{l}J \| c \\
\simeq 0.1\end{array}$ & 0.53 & 125 & 155 & 0.20 & 0.54 & 77.6 & 45.1 & $\cdot \cdot$ & 28.1 \\
\hline 2 & $\begin{array}{c}J \| b \\
=0.2\end{array}$ & 0.53 & 230 & 110 & 0.59 & 1.44 & 77.2 & 45.9 & 21.1 & 25.9 \\
\hline Chen et al. & $\begin{array}{l}J \| c \\
\simeq 2.0\end{array}$ & 0.52 & 165 & 150 & $\sim 0.4$ & $\sim 0.7$ & 63 & 40 & -18 & -18 \\
\hline $\begin{array}{l}\text { Rauchschwalbe } \\
\text { et al. }\end{array}$ &.. & 0.49 & $\cdot \cdot$ & $\cdots$ & $\cdots$ & . . & 60 & 40 & $\sim 19$ & $\sim 19$ \\
\hline
\end{tabular}

which cross, can arise only in the presence of an $l>0$ pairing interaction. There also may be additional information in the angular dependence of $H_{c 2}$ in the basal plane. Burlachkov and Gorkov ${ }^{7}$ have calculated the critical fields for anisotropic states possible for various crystal symmetries. They find that no changes should be observed when the field is rotated in the basal plane of a hexagonal crystal. Our measurements, shown in Fig. 2, are consistent with this prediction. It is possible, however, that there may be delicate mechanisms present which are washed out by our warming the sample above $T_{c}$ while top unloading to change the orientation of the samples.

The crossing of the curves for the critical field is not confined to the two perpendicular directions shown in Fig. 1. We have followed the disapperance of the anisotropy through the crossing point at various intermediate angles. These data are shown explicitly in Fig.

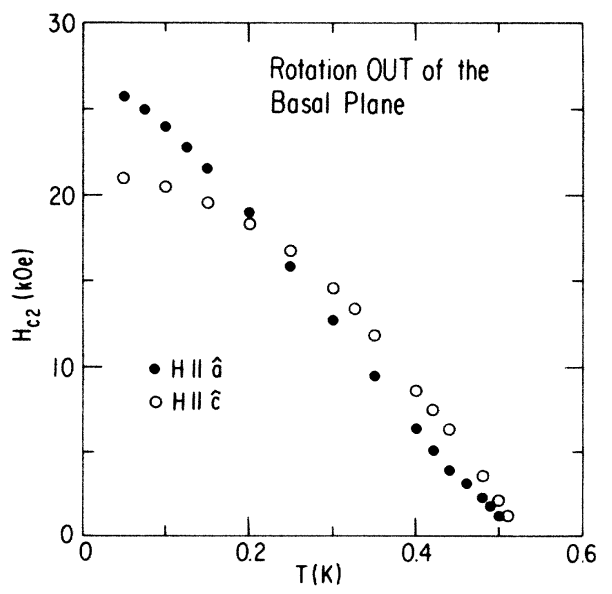

FIG. 1. The upper critical field, $H_{c 2}$, in (filled circles) and perpendicular to (open circles) the basal plane. The current is along $\hat{b}$ and always perpendicular to $H$. The curves for intermediate angles also cross at $T \sim 200 \mathrm{mK}$.
3 , where the lines drawn are guides to the eye. Note the change in the sense in which the critical field varies as a function of angle, as one goes through the crossing point. This is a new result which we are unable to understand within the framework of any of the present-day theories and it should place important constraints on future work.

Angular dependence similar to that shown in Fig. 3 was also studied near $T_{c}$. In Fig. 4, we plot the slope near $T_{c}$ obtained at various angles by fitting of a straight line to the data points above $450 \mathrm{mK}$. We have analyzed these results near $T_{c}$ using two models, one for isotropic pairing and the other which considers an anisotropic $p$-wave pairing interaction. Both these models are in the clean limit, which should be applicable to our samples as $l \gg \xi$.

In the standard WHH approach, the anisotropy in $H_{c 2}$ near $T_{c}$ arises basically from the angular depen-

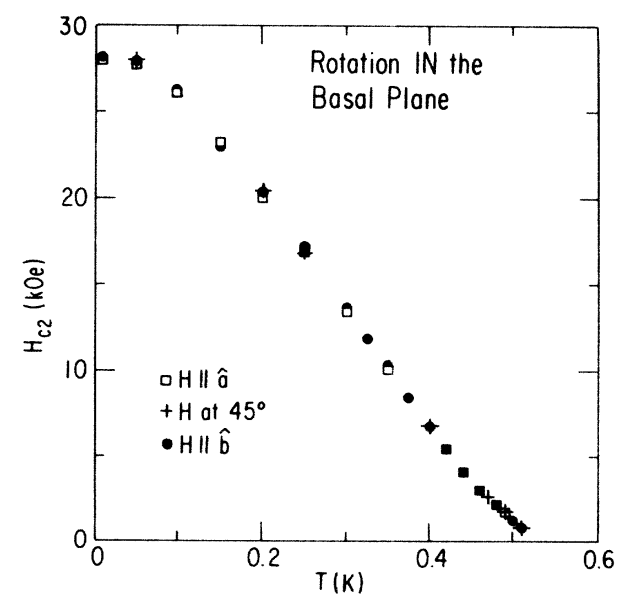

FIG. 2. The upper critical field, $H_{c 2}$, as a function of temperature appears independent of angle in the basal plane, a probable consequence of $\mathrm{UPt}_{3}$ 's hexagonal symmetry. The current is along $\hat{c}$ and always perpendicular to $H$. 


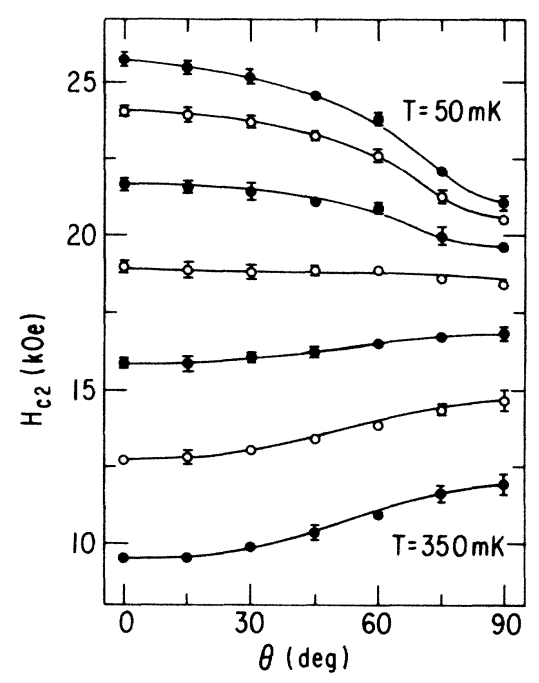

FIG. 3. The variation of $H_{c 2}$ with the angle $\theta$ out of the basal plane in $50 \mathrm{mK}$ increments for $50 \mathrm{mK} \leqslant T \leqslant 350 \mathrm{mK}$. The ansiotropy changes sense at $T \sim 200 \mathrm{mK}$. The solid lines are guides to the eye.

dence of the effective mass. ${ }^{10}$ For a hexagonal crystal this dependence is given by ${ }^{11}$

$$
H_{c 2}(\theta)-\left(\sin ^{2} \theta+\epsilon^{2} \cos ^{2} \theta\right)^{-1 / 2},
$$

where $\epsilon^{2}=m_{\perp} / m_{\|}$is the ratio of the effective masses perpendicular and parallel to the $c$ axis. The solid line in Fig. 4 is a least-squares fit by such an expression, which yields $m_{\perp} / m_{\|}=1.7$. This value is comparable with the results of susceptibility measurements. ${ }^{12}$

Hirschfeld and Sauls ${ }^{13}$ recently have obtained expressions for a $p$-wave superconductor with a uniaxial Fermi surface, considering weak anisotropy in both the pairing interaction and the effective mass. For the polar phase they find

$$
\begin{aligned}
H_{c 2}(\theta)-\sin ^{2} \theta & \left(1-\xi \cos ^{2} \theta\right) \alpha_{\|} \\
& +\cos ^{2} \theta\left(1+\frac{1}{2} \xi\left[\sin ^{2} \theta-\cos ^{2} \theta\right]\right) \alpha_{\perp},
\end{aligned}
$$

where $\xi=\left(m_{\perp}-m_{\|}\right) / m_{\|}$, and $\alpha_{\|}$and $\alpha_{\perp}$ measure the strength of the pairing interaction parallel and perpendicular to the $c$ axis, respectively. The dashed line in Fig. 4 is a result of fitting the experimental results to the polar state. We obtain the best-fit values $\xi=0.45$ and $\alpha_{\|} / \alpha_{\perp}=1.3$.

In considering both these models we have neglected several effects. Spin-orbit coupling is known to be important in heavy-mass superconductors. If there is unusual pairing, then the spin-orbit coupling can mix different angular momentum states. Scharnberg and Klemm, ${ }^{8}$ however, argue that spin-orbit coupling essentially can be neglected for crystals of hexagonal symmetry. In addition, although the zero-temperature critical field is not Pauli limited, ${ }^{2}$ the spin paramagne-

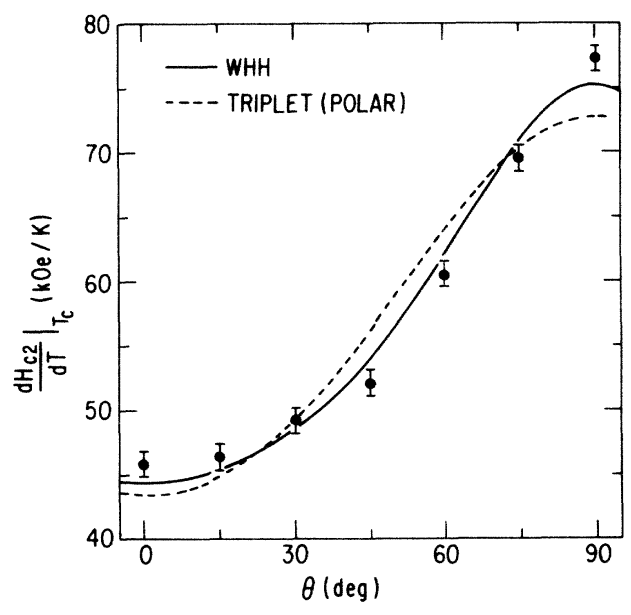

FIG. 4. The variation of the slope near $T_{c}$ with the angle out of the basal plane. The solid line is a fit by the standard isotropic model and the dashed line by a $p$-wave model, polar phase (see text).

tism could affect anisotropy near $T_{c}$. It is conceivable that incorporating these processes in the above theories will improve agreement with the experimental results near $T_{c}$.

We can only speculate at the mechanisms causing the $H_{c 2}$ curves to cross at low temperature. Recent neutron-scattering studies ${ }^{14}$ of single crystals of $\mathrm{UPt}_{3}$ indicate the presence of large antiferromagnetic spin fluctuations, restricted to the basal plane. The anisotropy in these fluctuations may be related to our observations of anisotropy in the upper critical field. If the pairing is triplet and if the correlation is ferromagnetic in nature, then the suppression of antiferromagnetic fluctuations by the external field should enhance $H_{c 2}$ in the basal plane, as observed. In addition to influencing the spin fluctuations, the magnetic field could have a perturbing effect on several other parameters in the superconducting state. Since the values for $H_{c 2}$ at low temperatures are extremely large, the magnetic energy is an appreciable fraction of the characteristic energy scale set by the "coherence temperature" 15 in $\mathrm{UPt}_{3}$. In such a situation there can be a significant modification of the electron $g$ factor, the spin-orbit coupling energy, and the energy gap. ${ }^{16}$

The fact that we observe the crossing of $H_{c 2 \perp}$ and $H_{c 211}$, whereas previous results indicated no such behavior down to $150 \mathrm{mK}$, may reflect different quality samples. On the other hand, it has been suggested ${ }^{13}$ that the curvature observed near $T_{c}$ in the work of Chen et al. $^{2}$ is due to the orienting effect of their larger current density on the order parameter. A similar effect could have perturbed the low-temperature behavior.

In summary, we find that as a function of angle out of the basal plane, the larger the critical field slope 
$d H_{c 2} / d T$ at $T_{c}$, the smaller the limiting value $H_{c 2}(T \rightarrow 0)$. At $T \approx 200 \mathrm{mK}, H_{c 2}$ is independent of angle. We also find that $H_{c 2}(T)$ is insensitive to rotation in the basal plane, which appears to be a manifestation of $\mathrm{UPt}_{3}$ 's hexagonal symmetry.

We thank S. B. Field for technical help and we have benefitted from conversations with G. Crabtree, R. A. Klemm, K. Levin, B. Patton, D. Rainer, J. P. Rodriguez, and J. A. Sauls. The work at The University of Chicago was supported by the National Science Foundation under Grant No. DMR 83-51992. The work at Argonne National Laboratory was supported by the $\mathrm{U}$. S. Department of Energy under Contract No. W-31109-ENG-38. One of us (T.F.R.) acknowledges receipt of an Alfred P. Sloan Research Fellowship.

(a) Permanent address: Physics Department, The University of Virginia, Charlottesville, VA 22901.

${ }^{1}$ G. R. Stewart, J. Appl. Phys. 57, 3049 (1985).

2J. W. Chen, S. E. Lambert, M. B. Maple, Z. Fisk, J. L. Smith, G. R. Stewart, and J. O. Willis, Phys. Rev. B 30, 1583 (1984); J. O. Willis, Z. Fisk, J. L. Smith, J. W. Chen, S. E. Lambert, and M. B. Maple, in Proceedings of the Seventeenth International Conference on Low-Temperature Physics, edited by U. Eckern, A. Schmid and W. Weber (North-Holland, Amsterdam, 1984), p. 245; U. Rauchschwalbe, U. Ahlheim, F. Steglich, D. Rainer, and J. J. M. Franse, Z. Phys. B 60, 379 (1985).

${ }^{3}$ D. Jaccard, J. Floquet, P. Lejay, and J. L. Tholence, J.
Appl. Phys. 57, 3082 (1985).

${ }^{4}$ D. J. Bishop, C. M. Varma, B. Batlogg, E. Bucher, Z. Fisk, and J. L. Smith, Phys. Rev. Lett. 53, 1009 (1984); V. Muller, D. Maurer, E. W. Scheidt, Ch. Roth, K. Luders, E. Bucher, and H. E. Bommel, Solid State Commun. 57, 319 (1986).

${ }^{5}$ B. S. Shivaram, Y. H. Jeong, T. F. Rosenbaum, and D. G. Hinks, Phys. Rev. Lett. 56, 1078 (1986).

${ }^{6}$ C. J. Pethick and D. Pines, to be published; P. Hirschfeld, D. Vollhardt, and P. Wölfle, to be published; S. Schmitt-Rink, K. Miyake, and C. M. Varma, to be published.

${ }^{7}$ L. P. Gorkov, Pis'ma Zh. Eksp. Teor. Fiz. 40, 351 (1984) [JETP Lett. 40, 1155 (1984)]; L. I. Burlachkov and L. P. Gorkov, to be published.

${ }^{8}$ K. Scharnberg and R. A. Klemm. Phys. Rev. Lett. 54, 2445 (1985); R. A. Klemm and K. Scharnberg, J. Magn. Magn. Mater. 54-57, 381 (1986).

${ }^{9}$ See, for example, Ref. 1, p. 3052.

${ }^{10}$ E. Helfand and N. R. Werthamer, Phys. Rev. 147, 288 (1966); L. P. Gorkov and T. K. Melik-Barkhudarov, $\mathrm{Zh}$. Eksp. Teor. Fiz. 45, 1493 (1963) [Sov. Phys. JETP 18, 1031 (1964)].

${ }^{11}$ M. Decroux and O. Fischer, in Superconductivity in Ternary Compounds $I$, edited by $\mathrm{M}$. B. Maple and O. Fischer (Springer-Verlag, Berlin, 1982), p. 57.

${ }^{12}$ A. de Visser, J. J. M. Franse, and A. Menovsky, J. Magn. Magn. Mater. 43, 43 (1984).

13P. J. Hirschfeld and J. A. Sauls, to be published.

${ }^{14} \mathrm{G}$. Aeppli, A. Goldman, G. Shirane, E. Bucher, and M.-Ch. Lux-Steiner, to be published.

${ }^{15}$ Z. Fisk, H. R. Ott, T. M. Rice, and J. L. Smith, Nature (London) 320, 124 (1986).

16M. Tachiki, T. Koyama, and S. Takahashi, Physica (Amsterdam) 132B, 57 (1985). 